\title{
(杂)芳烃 $\mathbf{C}-\mathbf{H}$ 键的电化学氧化胺化的新进展
}

\author{
曾程初* \\ 北京工业大学生命科学与生物工程学院, 北京 100124 \\ *通讯作者, E-mail: zengcc@bjut.edu.cn
}

收稿日期: 2018-09-03; 接受日期: 2018-09-04; 网络版发表日期: 2018-09-21

(杂)芳烃 $\mathrm{C}-\mathrm{H}$ 键的氧化胺化形成新的 $\mathrm{C}-\mathrm{N}$ 键在 有机化学、药物化学以及材料科学中具有重要的意义. 该反应通常在化学计量氧化剂的作用下，通过金属 催化氧化来实现. 化学计量氧化剂的使用, 将不可避 免地产生大量的废物, 也可能引起产物的过氧化, 导 致发生其他副反应，产率降低.

电化学方法通过对有机分子施加一定的电场力, 使其选择性地与电极间发生电子的得失, 从而实现 有机分子的还原和氧化. 有机电合成由于采用电子 作为反应试剂, 避免了化学氧化还原剂的使用, 因而 是一种环境友好的有机合成方法 ${ }^{[1]}$.

从原理上说, 芳烃 $\mathrm{C}-\mathrm{H}$ 的直接电化学氧化胺化 可以经由含氮化合物对电氧化形成的芳烃阳离子自 由基的原位亲核加成来实现. 然而, 由于芳环被胺基 取代后生成的胺基化产物的氧化电位比对应的芳烃 原料的氧化电位更低, 胺基化产物更易在电极表面 氧化, 常常得不到产物或者电流效率极低, 因此, (杂) 芳烃 $\mathrm{C}-\mathrm{H}$ 的直接电化学氧化胺化面临着巨大的挑战. 针对此问题, 2013 年日本京都大学的 Yoshida 等 ${ }^{[2]}$ 将 芳烃阳离子自由基的产生与胺基化产物的形成分解 为电反应和化学转化两个过程: 先在过量吡啶存在 下的双室电解槽中进行芳烃的电化学氧化, 这样阳 离子自由基一经形成即被吡啶捕获, 形成稳定的吡 啶盐中间体. 由于该吡啶盐带正电且氧化电位高, 过 氧化得以抑制。接着，在哌啶的作用下，吡啶盐中间 体开环裂解, 得到取代苯胺(图 1). 该方法可以有效
地使芳环 $\mathrm{C}-\mathrm{H}$ 转化为 $\mathrm{C}-\mathrm{NH}_{2}$. 遗憾的是该方法的底 物范围有限, 仅适用于苯甲醚等富电子的芳烃, 同时 $\mathrm{NH}_{2}$ 的引入缺少位置选择性, 有时会得到位置异 构体。

最近, 上海有机所的梅天胜课题组 ${ }^{[3]}$ 将金属催化 的 $\mathrm{C}-\mathrm{H}$ 键官能团化和电氧化相结合, 实现了铜催化 的(杂)芳烃与仲胺间的电化学 $\mathrm{C}\left(\mathrm{sp}^{2}\right)-\mathrm{H}$ 胺化反应. 其 基本思路是利用 $\mathrm{Cu}(\mathrm{III})$ 来进行芳环 $\mathrm{C}-\mathrm{H}$ 的氧化胺化, 同时在电催化剂 $\mathrm{Med}_{\mathrm{red}}$ 的存在下, 间接电氧化实现 $\mathrm{Cu}$ (III)的再生(图 2).

为此, 选择 $N$-苯基吡啶甲酰胺和哌啶的氧化偶 联为模型反应, 以 $n-\mathrm{Bu}_{4} \mathrm{NI}(50 \mathrm{~mol} \%)$ 和 $\mathrm{Cu}(\mathrm{OTf})_{2}(10$ $\mathrm{mol} \%$ )为电催化剂, 在单室电解槽中室温下恒电流电 解 (3 mA), 可以得到分离产率为 $86 \%$ 的目标产物 $3 \mathbf{a}$. 控制实验的结果表明, 催化剂 $\left(n-\mathrm{Bu}_{4} \mathrm{NI} 、 \mathrm{Cu}(\mathrm{OTf})_{2}\right)$ 、 碱(KOPiv)和电流是必不可少的. 该方法具有很好的

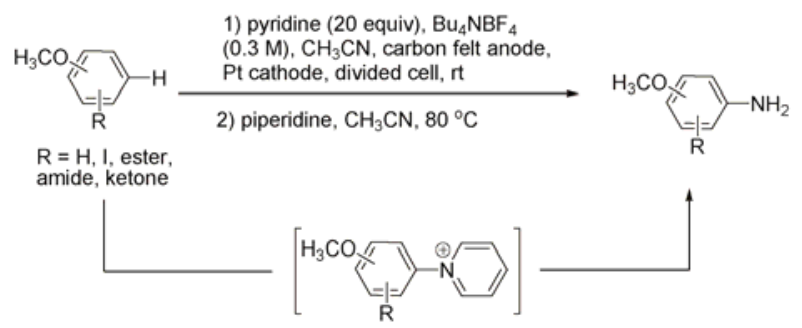

图 1 富电子芳烃的电化学氧化胺化 ${ }^{[2]}$

Scheme 1 Electrochemically oxidative amination of electron-rich arenes [2].

引用格式: Zeng C. New advances in the C-H amination of (hetero)arenes via electrochemical oxidation. Sci Sin Chim, 2018, 48: 215-217, doi: $10.1360 /$ N032018-00202 


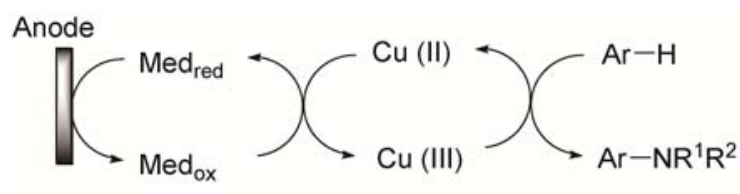

图 2 芳烃电化学碳-氢键胺化的双催化剂 ${ }^{[3]}$

Scheme 2 Dual catalysts for the electrochemical C-H amination of arenes [3].

官能团耐受性, 苯环上不仅可以是供电子的烷基、烷 氧基、硫醚等, 也可是缺电子的卤素、 $\mathrm{CF}_{3}$ 、酯基等, 甚 至羟甲基或碘等分别易于发生电氧化和电还原的基 团也适用. 更重要的是, 吡啶、喹啉环等在合成化学 和生物活性中具有重要应用的杂环也可发生胺基化 反应, 且选择性地在氮杂环氮原子的邻位发生(图 3). 遗憾的是氮亲核试剂的范围受限, 只适用于环状仲 胺, 而链状仲胺或伯胺未能与 $N$-苯基吡啶甲酰胺发 生氧化胺化反应.

该工作对有机电合成工作者来说具有非常重 要的参考价值. 以下从电化学合成的角度谈谈我的 认识.

首先，该反应首次实现了铜催化的(杂)芳烃与仲 胺间的电化学 $\mathrm{C}_{\mathrm{sp} 2}-\mathrm{H}$ 键的胺化. 2017 年以来, 金属催 化的 C-H 键官能团化和电化学氧化的结合受到关注, 如梅天胜、Ackermann、雷爱文以及徐海超等教授都 分别报道了他们在此领域内的工作, 而且 Ackermann 等 ${ }^{[4]}$ 和雷爱文教授最近也实现了 $\mathrm{Co}$ 催化的 $\mathrm{C}-\mathrm{H}$ 键官 能团化, 但金属催化的电化学 $\mathrm{C}-\mathrm{H}$ 键的官能团化主 要涉及 $\mathrm{Pd} 、 \mathrm{Ru}$ 和 $\mathrm{Rh}$ 等贵金属, 且反应常在双室电 解槽中进行. 此外, 现有报道的例子中过渡金属催化 剂的再生主要是通过直接电氧化实现的. 本文采用 便宜易得的碘离子为电催化剂, 通过间接电氧化实 现 $\mathrm{Cu}(\mathrm{III})$ 的再生. 这就意味着也可能使用其他廉价 金属, 通过电化学手段产生丰富的可变价态来进行 $\mathrm{C}-\mathrm{H}$ 键的官能团化. 这不仅使金属催化的 $\mathrm{C}-\mathrm{H}$ 键的 官能团化中金属催化剂的种类得到拓展, 也使电有 机合成的技术得到更广泛的应用.

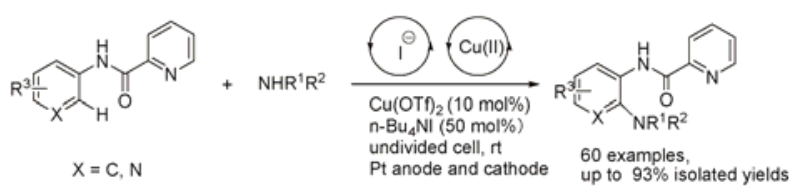

图 3 铜催化芳烃的电化学碳-氢键胺化

Scheme 3 Copper-catalyzed electrochemical C-H amination of arenes.
其次, 该文在电合成方法选择的思路方面将给 读者以启示. 金属离子催化的电有机合成面临两方 面的问题. 一是阳极氧化形成的高氧化态金属离子 极易在阴极被还原, 且氧化电位越高越易还原, 这就 要求电解反应在双室电解槽中进行; 二是组成氧化 还原电对的金属离子必须有一定的化学稳定性, 这 一般通过外加配体形成配合物来稳定. 该文中巧妙 地通过 $N$-苯基吡啶甲酰胺与铜离子的络合作用, 一 方面稳定了铜离子, 同时, 配合物的形成调控并降低 了该电对的氧化还原电位, 从而使 $\mathrm{Cu}(\mathrm{II})$ 可在更低的 电位下被氧化. 例如, $\mathrm{Cu}(\mathrm{II})$ 氧化为 $\mathrm{Cu}(\mathrm{III})$ 的起始电 位为 $2.42 \mathrm{~V}(\mathrm{vs} . \mathrm{Ag} / \mathrm{AgCl})$. 当 $\mathrm{Cu}(\mathrm{II})$ 与底物 $N$-芳基吡 啶甲酰胺形成配合物时, 中心 $\mathrm{Cu}(\mathrm{II})$ 离子的起始电位 负移到 $1.75 \mathrm{~V}$, 同时在吗啉的存在下, 能够进一步降 低到 $1.51 \mathrm{~V}$. 此起始电位值比底物 $N$-芳基吡啶甲酰 胺 $(2.06 \mathrm{~V})$ 和吗啉 $(1.61 \mathrm{~V})$ 在相同条件下的起始电位 值更低, 由此保证了电解发生时, 是中心 $\mathrm{Cu}(\mathrm{II})$ 离子, 而不是芳基吡啶甲酰胺和吗啉被氧化. 尽管如此, 该 配合物中心离子 $\mathrm{Cu}(\mathrm{II})$ 的电位也与对应的产物 3a 的 电位接近, 甚至略高 $(1.51 \mathrm{~V}$ vs. $1.44 \mathrm{~V})$. 因此, 当控 制在配合物中心离子 $\mathrm{Cu}(\mathrm{II})$ 的氧化电位处进行电解时, 将不可避免地会导致产物的过氧化分解. 针对此问 题, 作者加入另一电位更低的 $n-\mathrm{Bu}_{4} \mathrm{NI}$ 为电催化剂. 碘离子为单原子离子不会分解, 而且与常见的有机 电催化剂相比(四甲基哌啶氮氧化物(TEMPO)、2,3二氯-5,6-二氧对苯醌(DDQ)、醌、三芳胺和三芳基咪 唑等), 在溶液中的扩散速率和阳极间的非均相电子 转移速率大、过电位小, 从而使其与阳极间的非均相 电子转移不再是决速步骤. 在电化学反应中, 卤素离 子为电催化剂时一般是通过内层电子转移 (inner sphere electron transfer)机制来发挥作用, 此时虽然碘 离子和配合物中心 $\mathrm{Cu}(\mathrm{II})$ 的电位相差较大 $(0.9 \mathrm{~V}$ vs. $1.51 \mathrm{~V})$, 两者间的电子转移仍然可以发生, 从而使得 $\mathrm{Cu}(\mathrm{II})$ 被阳极氧化原位生成的活化态碘氧化为 $\mathrm{Cu}(\mathrm{III})$ 成为可能.

此外, 该文采用循环伏安法 $(\mathrm{CV})$ 和控制电位电 解库仑法研究反应的机理和分析实验结果很有特色. 本文采用反应动力学实验、动力学同位素效应、自由 基抑制实验以及电分析方法对反应机理进行了深入 细致的研究, 表明该反应很可能经历单电子转移过 程. 采用循环伏安法首先测量了两种反应底物 $(N$ - 苯 基吡啶甲酰胺和哌啶)、两种催化剂(铜离子和碘离子) 
和相应产物的氧化电位, 特别是起始电位值. 接着分 析了 $\mathrm{Cu}(\mathrm{II})$ 分别与两种反应底物形成配合物后起始电 位的变化, 由此表明, 配合物的形成可以降低 $\mathrm{Cu}(\mathrm{II})$ 的氧化电位, 同时说明起作用的是 $\mathrm{Cu}(\mathrm{III})$. 在此基础 上, 采用循环伏安法分析了碘离子与 $\mathrm{Cu}(\mathrm{II})$ 间的均相 电子转移的可能性. 催化电流的出现进一步说明碘 离子可以作为电催化剂实现 $\mathrm{Cu}(\mathrm{III})$ 的再生. 为了比 较直接电解与间接电解的不同, 作者又进行了控制 电位电解实验. 当控制电位在 $1.3 \mathrm{~V}$ 电解时, 由于没 达到 $\mathrm{Cu}(\mathrm{II})$ 的放电电位, 阳极与配合物间的电子转移 不能发生, 也就检测不到产物 $\mathbf{3 a}$ 的形成; 当分别控 制电位在 1.4 和 $2.0 \mathrm{~V}$ 电解时, $3 \mathbf{a}$ 的产率也比较低, 只 有 $20 \%$ 和 $53 \%$. 其主要原因就是在接近或高于产物 氧化电位时电解, 产物会不可避免地发生部分氧化 分解. 与此相反, 在相同条件下, 以 $n-\mathrm{Bu}_{4} \mathrm{NI}$ 为电催 化剂时, 只需控制在 $0.8 \mathrm{~V}$ 时电解, $\mathbf{3 a}$ 的产率可达到 $78 \%$. 这一结果表明, 间接电解可以在更低的电位下
获得更好的化学产率. 需要指出的是, 该工作在进行 电分析时, 只比较了起始电位的不同, 如果也能够关 注其对应的电流值的变化将使该分析结果更严谨. 同时, 如果能够采用化学方法制备相关配合物并研 究其电化学性质将有助于加深对机理的认识.

总之, 梅天胜课题组实现了首例铜催化的电氧 化促进的芳烃碳氢键胺化反应. 该反应在室温条件 下进行, 不需要使用带有隔膜的电解池, 因而为芳胺 的合成提供了高效、经济、环境友好的途径. 此外, 该 方法虽然使用昂贵的铂电极, 根据在以卤素离子为 媒介的电化学氧化方面的经验, 以便宜且易于加工 的其他电极材料代替铂是可能的. 而通过电化学工 程的手段又可能进一步减少 $n-\mathrm{Bu}_{4} \mathrm{NI}$ 的用量. 如果能 够解决这两个问题, 考虑到该方法采用适合于工业 化生产的恒电流电解技术和无隔膜电解槽, 又不需 外加导电盐等特点, 相信该方法将为芳胺的电化学 工业生产奠定坚实的基础.

\section{参考文献}

1 Lund H, Hammerich O. Organic Electrochemistry. 4th ed. Marcel: Dekker, Inc., 2001

2 Morofuji T, Shimizu A, Yoshida J-I. J Am Chem Soc, 2013, 135: 5000-5003

3 Yang Q-L, Wang X-Y, Lu J-Y, Zhang L-P, Fang P, Mei T-S. J Am Chem Soc, 2018, doi: 10.1021/jacs.8b07380

4 For a review, see: Sauermann N, Meyer T H, Qiu Y, Ackermann L. ACS Catal, 2018, 8: 7086-7103 